Supporting this, improvements were sustained at 2-year. $\mathrm{HbA} 1_{c}$ at 1-year is a useful barometer for the impact of changes to services.

\section{British Society for Rheumatology}

\section{RHEUM TO INSPIRE: A MEDICAL STUDENT PERSPECTIVE ON THE IMPACT OF COVID-19 ON MEDICAL EDUCATION AND CHILD HEALTH}

${ }^{1}$ Heather Keys, ${ }^{2}$ Anne-Marie McMahon, ${ }^{2}$ Daniel Hawley, ${ }^{2}$ Abhay Shivpuri, ${ }^{2}$ Rachel Tattersall. ${ }^{1}$ University of Sheffield; '² Sheffield Children's Hospital

10.1136/archdischild-2021-rcpch.526

Background When my Medical School announced my elective would be cancelled, and restricted to the local area, I was devastated. Months of planning which country to visit, medical opportunities there and making a holiday out of it were all lost. I had to quickly make a new plan. With no ideas for my future career, I considered specialities I had previously enjoyed: Paediatrics and Rheumatology.

Objectives I hope to provide an alternative perspective of the challenges and opportunities faced by Medical students and Child Health.

Methods With a large tertiary Children's Hospital close by, I contacted the Paediatric Rheumatology department to enquire. I reflect now, eight weeks after starting my Paediatric Rheumatology elective, having found my future career.

Results With the COVID-19 pandemic came a new style of working. Consultations via telephone and video link became commonplace, a frustrating concept being it is difficult to learn from these without face-to-face interaction. There was the additional issue of room space. Often face-to-face consultations or ward rounds were limited to a small number of essential staff, leaving no capacity for me to join. This new style of working however did appear to work for some patients, especially those who would normally have to travel far. Children whose arthritis was stable, or anxious parents insistent on shielding appeared happy with telephone appointments. Whilst periodic examinations are important, I feel there is scope for technology being continued for consultations post-pandemic, especially in stable, well children and those who travel far.

I did however witness the disruption COVID-19 has caused, especially on waiting times. Three month follow-ups became six months plus. Many children presented with worsening arthritis and increasingly problematic symptoms. Some had stopped their medication, either through parental fear of immunosuppression and COVID-19, side effects, or lack of motivation. Without regular follow-ups, these remained undetected for longer than previously. The increased number of arthritis flares I witnessed was supported by a service evaluation I conducted of steroid use prior to and during the pandemic. Preliminary results appear to show more 'fire-fighting' of flares during lockdown, and poorer outcomes. Lockdown has provided a unique opportunity to observe the need for a 'zero tolerance' approach to arthritis, something which should be imperative moving forward.

With the pandemic, brought Paediatric Multisystem Inflammatory Syndrome Temporally associated with COVID-19 (PIMS-TS). This new syndrome added extra challenges and pressures to the Rheumatology team. There were critically unwell children with long admissions, added to the backlog of usual work. I witnessed an overworked and pressured team, who were still welcoming and advocating for their patients. This is something I admire and the team were clinicians I aspire to be.

Conclusions Overall, I have witnessed many challenges faced by children and their families in this pandemic. I have seen opportunities moving forward, for the integration of technology and proactive approach to treating arthritis. For me personally, the pandemic has brought many challenges, with loss of loved ones and disruption to medical training, yet I thank the pandemic for giving me the opportunity to find my future career.

\section{Association of Paediatric Emergency Medicine}

\section{SUSPECTED CERVICAL SPINE TRAUMA IN THE PAEDIATRIC POPULATION - HOW VITAL IS THE ODONTOID PEG VIEW?}

${ }^{1}$ Jane Acty, ${ }^{2}$ Daniel Bradbury, ${ }^{2}$ Lisa Sabir, ${ }^{3}$ Derek Burke, ${ }^{2}$ Shammi Ramlakhan. ${ }^{1}$ Barnsley Hospital NHS Foundation Trust; ${ }^{2}$ Sheffield Children's Hospital; ${ }^{3}$ Gibraltar Health Authority

\subsection{6/archdischild-2021-rcpch.527}

Background Although rare, cervical spine injuries (CSI) can have devastating consequences. Use of c-spine CT in adult trauma is widespread. However, the desire to limit radiation exposure, along with recognition that CSI are uncommon in children, means that plain $\mathrm{x}$-rays are the more commonly used investigation in children with potential CSI.

NICE guidance recommends 3-view c-spine x-rays (anteroposterior, lateral and odontoid peg views - the latter in cooperative children who can open their mouth). However, risks of repeated exposure due to technical difficulties, and concerns that the view does not add diagnostic value raises questions about the added value of the odontoid view.

Objectives For children presenting to a Paediatric Major Trauma Centre (MTC), who required c-spine x-ray imaging, we aimed to:

- Estimate the incidence and type of CSI seen

- Determine the proportion with an adequate odontoid peg view, and the added value of repeat views.

- Estimate an age cut-off at which odontoid peg views may be pragmatically performed as routine.

Methods Data was extracted from the digital radiology database over a 5-year period for all children up to 16-years of age who had 3-view c-spine $\mathrm{x}$-ray series for trauma. Demographic data and outcomes including incidence of CSI were obtained.

Each series of images were reviewed by two blinded reviewers for quality and adequacy. Reviewers used quality criteria as defined by the European Guidelines on Quality Criteria for Diagnostic Radiographic Images in Paediatrics. Inadequate films were those that met less than $50 \%$ of the quality criteria.

Results 271 patients were included: 66 were aged $<10$ years (with 231 radiographs performed), and 205 children aged 
$\geq 10$ years (716 radiographs). $30 \mathrm{CTs}$ (70\% in children $\geq 10$ years) and 8 MRIs (all in children $\geq 10$ ) were obtained.

Overall, 3 children $(0.01 \%)$ had abnormalities - 2 undisplaced odontoid fractures (in an 8-year-old and a 12-year-old), and a C7 avulsion fracture (in a 14-year-old).

There were no significant differences in the quality of the lateral or AP radiographs in children $<10$ compared with $\geq 10$ years. However, significantly more (72.8\%; 95\%CI $62.2 \%-81.3 \%$ ) of the odontoid peg views in children $<10$ years were of inadequate quality, compared with those in children $\geq 10$ years $(50.2 \%$;95\%CI $43.8-56.6)$.

There was no significant improvement in the proportion of adequate quality views in children in whom additional views were obtained (to improve quality/adequacy), compared with those who had 3 views only.

Conclusions Although uncommon, upper CSI are still seen in young children. There is insufficient evidence to completely exclude the odontoid peg view from cervical spine imaging in paediatric trauma. However, a pragmatic approach to imaging which avoids unhelpful additional views is acceptable.

In children $<10$ years, the lateral and AP views should first be reviewed, and only if adequate, should an odontoid peg view be performed.

Additional views do not improve adequacy, hence inadequate initial views should prompt consideration of CT (after senior review and reassessment). Experience with injured children suggests some may have resolution of symptoms or be calmer, allowing more accurate senior assessment.

\section{Association of Paediatric Emergency Medicine}

\section{MENTAL HEALTH PRESENTATIONS TO A PAEDIATRIC EMERGENCY DEPARTMENT DURING A GLOBAL PANDEMIC}

${ }^{1}$ Helen Stewart, ${ }^{2}$ Lisa Sabir, ${ }^{2}$ Shammi Ramlakhan, ${ }^{2}$ Frances Yarlett. 'Sheffield Children's Hospital NHS Foundation Trust; ${ }^{2}$ Sheffield Children's NHS Foundation Trust

\subsection{6/archdischild-2021-rcpch.528}

Background Recent anecdotal reports suggests that more and more children are experiencing poor mental health as a result of multiple factors relating to the ongoing pandemic and subsequent school closures. We aimed to describe this change within mental health presentations at our own Paediatric Emergency Department. The department is located at a large children's hospital where, in 2019, we saw nearly 60,000 patients. Since March 2020 and the onset of the coronavirus pandemic, we have seen less than half that number.

Objectives To understand the impact of the coronavirus pandemic and school closures on the mental health of the population attending a paediatric emergency department.

Methods All patients under 16 who attended the emergency department with a mental health problem between 01/02/2019 and 31/01/2021 were identified. The number of presentations and admissions were analysed, as well as the index of multiple deprivation based on the patient's postcode.

Results Over the 24 month period we had 1431 mental health related attendances with a median age of 14 years. When looking at attendances by month, the mean number of presentations was 53.6 and the median, 61.5. Based on this data, we found that there has not been a significant increase or decrease in the number of attendances over the period studied, with the month with the most attendances being January 2020. It must be remembered that there has been a significant reduction in ED attendances overall and so the proportion of our patient population suffering from a mental health problem has increased.

The mean number of admissions over this time was 28.8 and the median 28.5. There has been an increase in admissions however this seems to have been a steady increase over the 24 months rather than a sudden upsurge during the pandemic, but again, this will equate to an increase in the proportion of admissions that are related to a mental health problem. Specific conditions, such as eating disorders, did show a large increase in numbers during the pandemic.

The vast majority of these patients are in the lowest decile, the 1 st, on the index of multiple deprivation. Rather than a gradual reduction in mental health problems as socioeconomic circumstances improve, there is a fairly consistent picture over the $3 \mathrm{rd}$ to 10th deciles, with a slightly higher incidence in the 2 nd. Conclusions Absolute numbers of presentations to an emergency department with mental health problems, and onward admissions into the hospital, have not increased significantly during the pandemic but the proportion of patients we see with mental health problems has. The majority of presentations are from sections of the population with the most challenging socioeconomic circumstances.

Our next step is to understand if the presentations we have seen have been more severe during the pandemic. Understanding the long term effects and identifying risk and resilience factors are vital for future planning of services and making these more accessible.

\section{Quality Improvement and Patient Safety}

\section{CAN ONLINE VIRTUAL INSTRUCTION DELIVER SUCCESS FOR RCPCH COVID-ADAPTED CLINICAL EXAMINATION CANDIDATES?... WE THINK SO}

Hannah Jane Davies, Sophie Constantinou. University hospital of UK

\subsection{6/archdischild-2021-rcpch.529}

Background Most doctors find examinations very stressful. In order to gain membership to the Royal College of Paediatrics, paediatricians are required to sit a clinical exam. Since November 2020, candidates have had to undertake a new COVID-Adapted Clinical exam involving new examination techniques and an online platform. We noticed the change caused increased anxiety and stress amongst our fellow trainees during an already challenging time.

Objectives To improve exam preparedness and wellbeing for trainees in Wales sitting the COVID-Adapted Clinical exam Methods We collaborated with the $\mathrm{RCPCH}$ examination team to gain insights into the new exam structure and expected standard. Prior to exam diets in November 2020 and February 2021 we provided 4-6 weeks of virtual teaching delivered via Microsoft Teams by registrars (ST4 and above) and Consultants. In November 2020, we organised the first COVIDAdapted Clinical Mock Exam in the UK using the online platform 'Zoom.' We wrote a range of simulated stations and utilised break out room sessions to facilitate the migration of 